\title{
Simulation Studies on Quasi-Bound States in the Continuum Introduced by Out-of-Plane Symmetry Breaking in Dielectric Metasurfaces
}

\author{
Received 9 December, 2021; revised 30 December, 2021; accepted 4 January, 2022
}

\begin{abstract}
Hyunsung Lim and Jeeyoon Jeong* (iD
Department of Physics and Institute of Quantum Convergence Technology, Kangwon National University, Chuncheon 24341, Republic of Korea
\end{abstract}

\section{*Corresponding author E-mail: peterjjy@kangwon.ac.kr}

\begin{abstract}
Metasurfaces with high quality factor resonances can be fabricated by breaking the symmetry of the constituent resonators, thereby converting symmetry-protected dark states to quasi-bound states in the continuum. As the quality factor is inversely proportional to the degree of the asymmetry, high quality factor metasurfaces typically comprise fine-tuned resonators requiring state-of-the-art lithographic technologies to fabricate. In this study, a method is proposed to create quasi-bound states in the continuum in dielectric metasurfaces without high-resolution lithography. The results show that quasi-bound states in the continuum can be formed by introducing a non-vertical sidewall in an array of cube resonators, such that the resonator symmetry breaks in an out-of-plane direction. The proposed method enables fabrication by controlling the dry etching angle without extensive use of high-resolution lithography. Using full-wave electromagnetic simulations and multipole analyses, high quality factor resonances are observed in the proposed metasurface structure, the connections of which to the quasi-bound states in the continuum are clarified. Our work enables fabrication of high quality factor metasurfaces with low cost and high controllability, and is expected to greatly benefit the field of enhanced light-matter interactions in nanophotonics.
\end{abstract}

Keywords: Metasurface, Quasi-bound states in the continuum, Quality factor, Lithography, Cube resonators, Symmetry breaking

\section{Introduction}

A metasurface is a two-dimensional array of resonators with subwavelength dimensions and periods, which can be engineered to have desirable properties absent in natural materials. Several photonic applications such as flat optics, sensors, and photodetectors are based on metasurfaces due to their flexibility in manipulating electromagnetic fields at the sub-wavelength scale [1-6]. In particular, dielectric metasurfaces can support Mie resonances with various types of multipole moments capable of efficiently boosting nanoscale light-matter interactions [7-9]. Such metasurfaces have been utilized to enhance photoluminescence, harmonic generation, and nonlinear mixing, etc. in optically active materials including III-V semiconductors and lowdimensional materials [10-14]. Further enhancement is possible by realizing resonances with high quality factors $(\mathrm{Q})$, which increase local photonic density of states. In metasurfaces, high quality factor resonances are typically realized by introducing an asymmetry into an otherwise symmetric unit cell. This distorts the symmetry-protected 'dark' Mie resonances and allow them to couple with the incident radiation, thereby converting the 'dark' bound states in the continuum (BIC) into quasi-bound states in the continuum (quasi-BIC) [15]. Various types of high-Q metasurfaces with asymmetric unit cells such as a dimer of ellipsoids, split ring resonators, distorted cubes, and double bar structures were reported to be extremely effective in enhancing light-matter interactions [16-19].

Metasurfaces with quasi-BIC are known to have a quality factor of resonance that is inversely proportional to the square of the asymme- try parameter. Therefore, high-Q resonances can be achieved through minimal adjustments using a symmetric resonator. For metasurfaces designed for visible or near-infrared wavelengths, the typical size of an individual resonator is of the order of $100-300 \mathrm{~nm}$ and introduced asymmetry must be of the order of a few tens of nanometers. This critical dimension requisite is extremely difficult to achieve with commonly used, scanning electron microscope (SEM)-based electron beam lithography (EBL) systems and generally require extremely expensive e-beam writers with acceleration voltage of $\sim 100 \mathrm{kV}$. Therefore, a high-Q metasurface that can be fabricated without expensive lithographic tools is expected to significantly benefit research on metasurfaces and enhanced light-matter interactions.

In this study, a fabrication method for high-Q metasurfaces operating at near-infrared wavelengths without employing a high-end e-beam writer. Typically, asymmetric dielectric metasurfaces are fabricated in a particular order [20]. First, a hard mask with an asymmetric pattern is created using EBL atop a dielectric film. Then, the asymmetric pattern is transferred to the underlying dielectric film in the process of dry etching. This standard process creates resonators with in-plane asymmetry. The proposed method begins with the creation of a hard mask with a large, symmetric pattern that can be easily handled with common SEM-based EBL systems (Fig. 1). Following this, the pattern is transferred to the film in the dry etching step, as in the conventional method. Subsequently, an additional dry etching is applied at a non-vertical angle, thereby creating non-vertical sidewalls and out-of-plane oriented asymmetry. The etching angle can be controlled either by tilting the loading surface of the handling wafer, or 
(a)

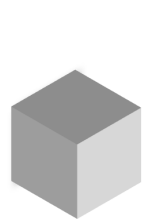

Symmetric

(b)

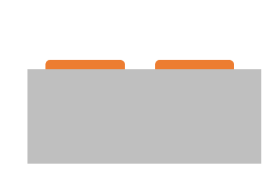

Hard mask Patterning w/ EBL

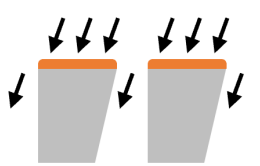

Angled dry etching

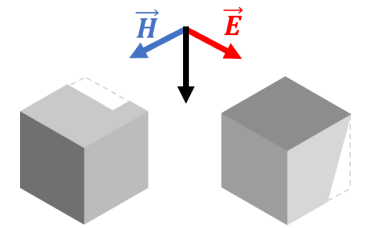

Asymmetry in-plane

\section{Asymmetry}

out-of-plane

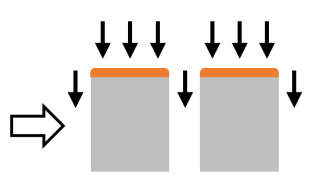

Anisotropic dry etching
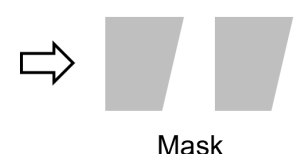

removal
Figure 1. (a) Symmetric and asymmetric resonators for dielectric metasurfaces. Most asymmetric resonators have their symmetry broken in-plane. By introducing a non-vertical sidewall to one of the sides of the resonator, it is also possible to make out-of-plane-asymmetric resonator. (b) Proposed fabrication method for the out-of-plane-asymmetric resonators. First dry etching step makes an array of perfectly symmetric resonators, and an additional, angled dry etching creates the out-of-plane asymmetry to the resonators.

by using etching facilities with angle controllability such as argon ion beam millers. Therefore, the metasurface with out-of-plane asymmetry can be practically realized but, nevertheless, remains unexplored. Finally, using full wave electromagnetic simulations, the optical properties of such metasurfaces are analyzed and the physical origin of their resonances is discussed.

\section{Methods}

For electromagnetic simulations, a python-based finite difference time domain (FDTD) method library called MEEP was employed [21]. The proposed metasurface comprised an array of silicon cubes with slightly non-vertical sidewalls along one side (Fig. 2). The length of each side of the cube $(l)$ was $300 \mathrm{~nm}$, and period of the array $(p)$ was $470 \mathrm{~nm}$ in both the $\mathrm{x}$ and $\mathrm{y}$ directions. Structural asymmetry was controlled by the amount of undercut $(g)$ which varied from 0 to $100 \mathrm{~nm}$. The two vertical sidewalls of the cubes were normal to the $x$ axis, and the non-vertical sidewall faces the y-direction. The wavelength window for the simulation was 1,000 to $1,500 \mathrm{~nm}$, considering that silicon is nearly lossless and exhibits a high refractive index of $\sim 3.6$. The simulation was run with a mesh size of $10 \mathrm{~nm}$, until the remnant electric field across the entire simulation volume dropped below $10^{-7}$ of the initial electric field. Incident radiation propagated in $z$ direction, with a $x$-polarized electric field and a $y$-polarized magnetic field. The radiation was launched $450 \mathrm{~nm}$ above the metasurface and was transmitted through the metasurface in the normal $(z$-) direction, and the transmitted radiation was collected by a flux monitor also placed $450 \mathrm{~nm}$ below the metasurface. Electric field distribution within the metasurface was also recorded using a separate near-field monitor. In addition, a separate simulation without any structure was performed to normalize the transmitted flux and electric field distributions. Perfectly matched layers were placed on either end of the $+z$ and $-z$ sides to preclude simulation artifacts in edge reflections.

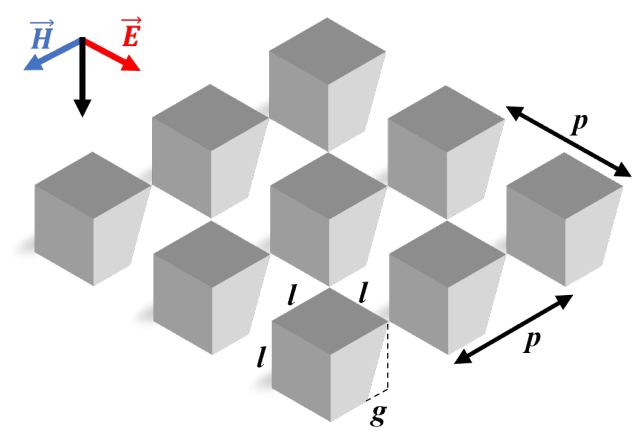

Figure 2. Structure of the dielectric metasurface under study. Here, $l=300 \mathrm{~nm}, p$ $=470 \mathrm{~nm}$, and $g$ varies from 0 to $100 \mathrm{~nm}$.

Post simulation, the Q-factor of the resonances was extracted by fitting the transmission spectra with the following Fano formula [22],

$$
t(\omega)=a \omega+b+c \times \frac{\left(q+\left(\omega-\omega_{0}\right) / \gamma\right)^{2}}{1+\left(\omega-\omega_{0}\right)^{2} / \gamma^{2}} .
$$

Here, $\omega$ is angular frequency, $a \omega+b$ represents the background spectrum, $c$ is the amplitude of the resonant mode, $q$ is the asymmetry parameter of the Fano resonance, and $\gamma$ is loss rate. Quality factor of the resonance can be calculated as $Q=\omega_{0} / 2 \gamma$. As quasi-BIC is essentially an interference of a broad resonance (continuum state) and a sharp resonance (bound state), we expect the line to be asymmetric rather than Gaussian or Lorentzian.

Mie modes contributing to the resonances are analyzed with multipole decomposition studies. From electric field distribution within the resonators, multipole moments can be calculated using the following equations $[20,23]$,

$$
\begin{aligned}
\vec{J}(\vec{r}) & =i \omega \epsilon_{0}(\epsilon-1) \vec{E}(\vec{r}), \\
\vec{p} & =\frac{1}{i \omega} \int \vec{J} d V, \\
\vec{m} & =\frac{1}{2 c} \int \vec{r} \times \vec{J} d V, \\
\vec{t} & =\frac{1}{10 c} \int\left[(\vec{r} \cdot \vec{J}) \vec{r}-2 r^{2} \vec{J}\right] d V, \\
Q E_{\alpha \beta} & =\frac{1}{i 2 \omega} \int\left[r_{\alpha} J_{\beta}+r_{\beta} J_{\alpha}-\frac{2}{3} \delta_{\alpha \beta}(\vec{r} \cdot \vec{J})\right] d V, \\
Q M_{\alpha \beta} & =\frac{1}{3 c} \int\left[[\vec{r} \times \vec{J}]_{\alpha} r_{\beta}+[\vec{r} \times \vec{J}]_{\beta} r_{\alpha}\right] d V .
\end{aligned}
$$

Here, $\vec{J}$ is displacement current density, $\vec{r}$ is position vector, $\epsilon_{0}$ is vacuum permittivity, $\epsilon$ is relative permittivity of dielectric material comprising the resonator, $\vec{E}$ is electric field, $c$ is the speed of light, and $\vec{p}, \vec{m}, \vec{t}, Q E$, and $Q M$ are electric dipole, magnetic dipole, toroidal dipole, electric quadrupole, and magnetic quadrupole moments, respectively. The integration is performed across the entire resonator volume. To compare the amplitudes of the scattered electric field from these multipoles, the electric or magnetic dipole moments, toroidal dipole or electric quadrupole moments, and magnetic quadrupole moment require additional factors of $\omega / c, \omega^{2} / c^{2}$, and $\omega^{2} / 2 c^{2}$ respectively.

\section{Results and discussion}

Figures 3(a) and 3(b) show the calculated transmission spectra of asymmetric $(g=100 \mathrm{~nm})$ and symmetric metasurfaces. Transmission 
(a)

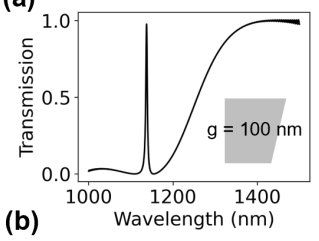

(b)

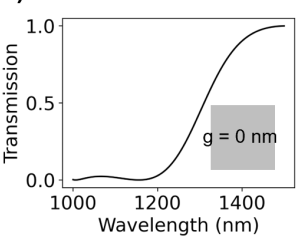

(c)

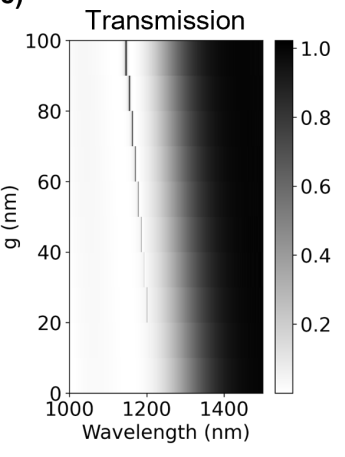

Figure 3. Transmission spectra of metasurfaces with (a) $g=100 \mathrm{~nm}$, and (b) $g$ $=0 \mathrm{~nm}$. (c) 2-dimensional map of transmission spectra of metasurfaces with different $g$ 's.

through a symmetric metasurface exhibits broad dispersion with nearzero transmission at $1,100 \mathrm{~nm}$ and a transmission value approaching unity at wavelengths above $1,300 \mathrm{~nm}$. In contrast, transmission spectra of the asymmetric metasurface exhibit additional sharp resonance with a transmission value approaching unity at $1,100 \mathrm{~nm}$. To study the dependence of the resonances on the asymmetry, all calculated spectra were plotted in a colormap, as shown in Fig. 3(c). A gradual red shift of spectral features is observed as $g$ decreases, owing to an increased overall volume of the resonator causing redshift in the Mie modes. More importantly, the resonance become sharper and finally disappears at the limit of $g=0$, corroborating the hypothesis that the resonances originate from quasi-BIC formed by symmetry breaking.

For a more quantitative analysis, Q-factors of the resonances were extracted using Eq. (1) and plotted in Fig. 4. The Q-factors increase divergently as $g$ approaches zero, implying the presence of quasi-BIC. Q-factors up to 7913 were observed at $g=20 \mathrm{~nm}$; although higher Q-factors were expected for smaller $g$ values, limited computational resources made it difficult to observe resonances for $g$ values below 20 $\mathrm{nm}$. Nevertheless, the results indicate that it is practically possible to incorporate quasi-BIC by introducing an out-of-plane asymmetry.

The electric field distribution of the resonant mode for a sample with $g=100 \mathrm{~nm}$ is shown in Fig. 5. A strong field enhancement up to a factor of 12 is observed, which becomes larger for resonances with a smaller $g$ and larger Q-factor (data not shown). Moreover, the electric field distribution describes a circular pattern in the $x-y$ plane, implying an existence of inductive displacement current and a corresponding excitation of a magnetic dipole oscillating the in $z$-direction. A pure electric or magnetic dipole is a 'dark' mode because it does not couple to external radiation incident in its oscillating direction. Therefore, the detection of such modes provides further proof of the quasi-BIC nature of the resonance, such that the out-of-plane magnetic dipole mode corresponds to the 'bound state.'

Further quantitative analysis of the nature of the resonance was performed through multipole decomposition studies using Eqs. (2)- (a)

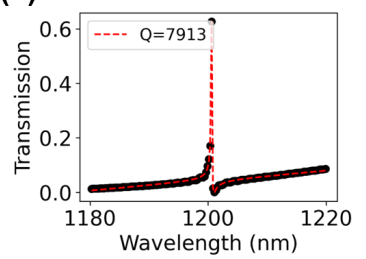

(b)

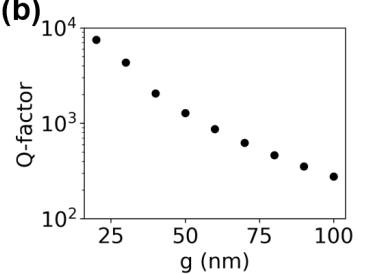

Figure 4. (a) Simulated transmission spectrum for $g=20 \mathrm{~nm}$ (black dot) and its Fano fitting curve (red dashed line), showing an extracted Q-factor of 7913. (b) Extracted Q-factors versus $g$. (a)

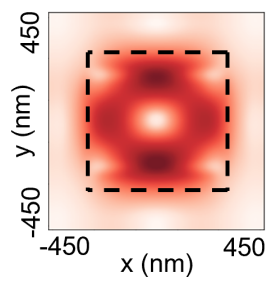

(b)

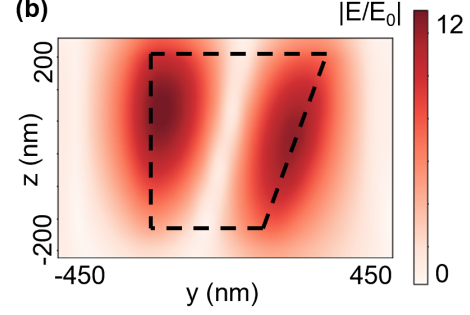

Figure 5. Electric field distribution for $g=100 \mathrm{~nm}$ sample. (a) $x-y$ plane field distribution at the middle height $(z=0 \mathrm{~nm})$ of the resonator. (b) $y-z$ plane field distribution at the middle $(x=0 \mathrm{~nm})$ of the resonator.

(7), the results for which are shown in Fig. 6. For a metasurface with perfectly symmetric resonators $(g=0 \mathrm{~nm})$, the dominant multipolar component for a broad transmission minimum near wavelength of $1,100 \mathrm{~nm}$ is found to be a $y$-oriented magnetic dipole $\left(m_{y}\right)$, along with similarly large $x$-oriented electric dipole $\left(p_{x}\right)$. When the out-of-plane symmetry is broken $(g=100 \mathrm{~nm}), m_{y}$ decreases considerably resulting in the emergence of $m_{z}$ with a scattering field amplitude enhanced by a factor greater than five. This implies that most of the energy induced in $m_{y}$ is transferred to $m_{z}$ and the inward coupled electromagnetic energy is stored owing to its inability couple directly to the normally incident radiation. Therefore, the resonance can be interpreted as a bound state $\left(m_{z}\right)$ weakly coupling to a continuum state $\left(m_{y}\right)$, creating a quasi-BIC with Q-factor inversely dependent on $g$.

Furthermore, the role of the undercut in the coupling of the bound and continuum states can be explained as follows. In a perfectly symmetric cube, $m_{z}$ incorporates an electric field that circulates in the $x-y$ plane, while in $m_{y}$, the electric field circulates in the $z-x$ plane. Despite the presence of a common electric field component $E_{x}$ between the two modes, the parity of the field is opposite in the upper and lower halves of the resonator for $m_{y}$. In other words, field overlap between the two modes perfectly cancel out, which means that no net energy transfers from $m_{y}$ to $m_{z}$ mode. Therefore, in a perfectly symmetric cube the $m_{z}$ mode cannot couple to incident radiation and remains a perfectly bound state [Fig. 7(a)]. In a resonator with undercut, the volumes of the upper and lower parts of the resonator differ, and $m_{y}$ is distorted such that two different parities of $E_{x}$ do not even out. This leads to a non-cancelling field overlap between $m_{y}$ and $m_{z}$ modes such that excitation of $m_{y}$ induces electromagnetic energy transfer into $m_{z}$ [Fig. 7(b)].

The aforementioned mechanism implies that the coupling between two modes could potentially be absent for a different polarization. If the incident magnetic field is polarized in the $x$ direction such that generated magnetic dipole moment is normal to the two vertical sidewalls, then the distortion of $m_{y}$ induced by the undercut primarily affects parities of $E_{z}$. As $m_{z}$ solely contains the $x$ and $y$ components of the electric field, the field overlap remains unaffected by the mode distortion and no coupling between $m_{y}$ and $m_{z}$ can be established [Fig. 7 (c)]. This is clearly illustrated in Figs. $7(\mathrm{~d})$ and $7(\mathrm{e})$, where transmission with cross-polarized incident radiation does not exhibit any
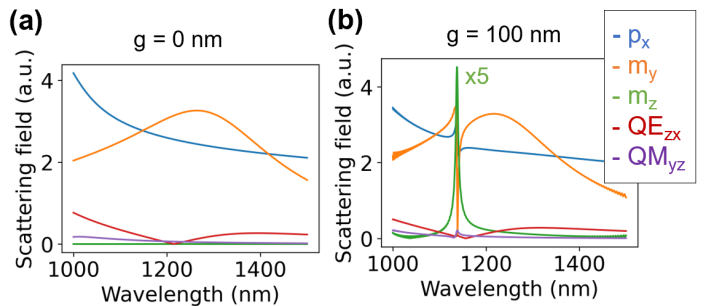

Figure 6. Multipole decomposition studies for samples with (a) $g=0 \mathrm{~nm}$ and (b) $g=100 \mathrm{~nm}$. In these structures, toroidal dipole moment $t_{x}$ is found to be very small and is omitted in the spectra. 


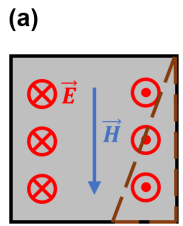

Bound state $\left(m_{z}\right)$

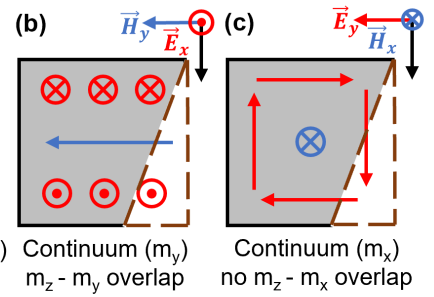

(e)

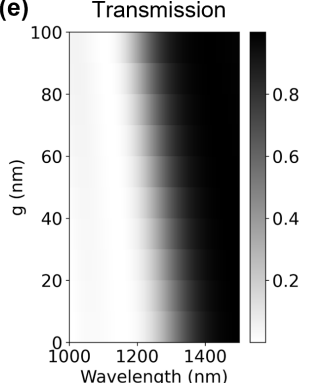

Figure 7. Polarization dependence of quasi-BIC and the role of undercut. (a) Schematic drawing of electromagnetic field distribution of $m_{z}$ mode. When an undercut is introduced (brown dashed line), distribution of $E_{x}$ and $E_{y}$ will be distorted as in Fig. 5. (b) Field distribution of $m_{y}$ mode, which will have distorted $E_{x}$ and $E_{y}$ distribution as well. Therefore, the undercut creates a common asymmetry in both $m_{y}$ and $m_{z}$, thereby coupling the two modes. (c) Field distribution of $m_{x}$ mode which will appear for a cross-polarized incident radiation. Here, the undercut mostly creates asymmetry in $E_{z}$ distribution, such that $m_{x}$ does not couple with $m_{z}$. (d) Simulation schematic for a cross-polarized incident radiation (top) and resulting transmission spectrum for $g=100 \mathrm{~nm}$ (bottom). (e) 2-dimensional map of transmission spectra under cross-polarized radiation, showing no sharp resonances.

high-Q resonances despite apparent symmetry breaking in the outof-plane direction. It is noteworthy, that even with cross-polarized light, quasi-BIC can be excited from different multipole modes with different symmetries, such as in-plane electric dipole and out-of-plane magnetic dipole modes, albeit in different wavelengths.

\section{Conclusions}

In this study, the formation of quasi-BIC induced by out-of-plane symmetry breaking was investigated in metasurfaces. A sharp resonance with a Q-factor inversely proportional to the degree of undercut was observed, which is strongly dependent on the polarization of incident radiation. Multipole decomposition studies revealed the detailed nature of the resonances and the role of undercut in establishing the quasi-BIC. The proposed method presents an additional tool for controlling the high-Q resonances which offers great tunability for metasurface applications. Furthermore, the proposed fabrication method of tilted dry etching can be applied to dielectric resonators that are considerably smaller or larger for operation in different wavelength ranges spanning from visible to far-infrared. While our calculation does not include effect of a substrate and may not fully reflect optical properties of metasurfaces in realistic systems, qualitative agreement may still be expected due to a relatively low refractive index of commonly used substrates $(\sim 1.52$ for glass) compared to that of the resonators $(\sim 3.6)$. Therefore, this study is expected to introduce several possibilities for application of high-Q metasurfaces in enhancing light-matter interactions in various semiconductors and low-dimensional quantum materials.

\section{Acknowledgements}

This project is supported by a National Research Foundation of Korea (NRF) grant funded by the Korean Government (MSIT:
NRF-2021R1C1C1010660) and a 2020 Research Grant from Kangwon National University. The authors would like to thank Editage (www.editage.co.kr) for English language editing.

\section{Conflict of Interest}

The authors declare no conflicts of interest.

\section{ORCID}

Jeeyoon Jeong

https://orcid.org/0000-0003-1708-0492

\section{References}

[1] M. Khorasaninejad, W. T. Chen, R. C. Devlin, J. Oh, A. Y. Zhu, and F. Capasso, Science 352, 1190 (2016).

[2] M. Khorasaninejad and F. Capasso, Science 358, eaam8100 (2017).

[3] R. Sarma et al., Appl. Phys. Lett. 113, 201101 (2018).

[4] L. Cong, Y. K. Srivastava, H. Zhang, X. Zhang, J. Han, and R. Singh, Light Sci. Appl. 7, 28 (2018).

[5] Y. Lee, S.-J. Kim, H. Park, and B. Lee, Sensors 17, 1726 (2017).

[6] T. Siday, P. P. Vabishchevich, L. Hale, C. T. Harris, T. S. Luk, J. L. Reno, I. Brener, and O. Mitrofanov, Nano Lett. 19, 2888 (2019).

[7] A. B. Evlyukhin, S. M. Novikov, U. Zywietz, R. L. Eriksen, C. Reinhardt, S. I. Bozhevolnyi, and B. N. Chichkov, Nano Lett. 12, 3749 (2012).

[8] O. Mitrofanov, F. Domenic, P. Kužel, J. L. Reno, I. Brener, U.-C. Chung, C. Elissalde, M. Maglione, and P. Mounaix, Proceeding of the Quantum Sensing and Nanophotonic Devices XII 9370 (San Francisco, CA, USA, February 8, 2015). 937005.

[9] Q. Zhao, J. Zhou, F. Zhang, and D. Lippens, Mater. Today 12, 60 (2009).

[10] T. Bucher et al., ACS Photonics 6, 1002 (2019).

[11] S. Liu, P. P. Vabishchevich, A. Vaskin, J. L. Reno, G. A. Keeler, M. B. Sinclair, I. Staude, and I. Brener, Nat. Commun. 9, 2507 (2018).

[12] S. Liu, G. A. Keeler, J. L. Reno, M. B. Sinclair, and I. Brener, Adv. Opt. Mater. 4, 1457 (2016).

[13] K. Schneider, P. Welter, Y. Baumgartner, H. Hahn, L. Czornomaz, and P. Seidler, J. Light. Technol. 36, 2994 (2018).

[14] S. Liu et al., Nano Lett. 16, 5426 (2016).

[15] K. Koshelev, S. Lepeshov, M. Liu, A. Bogdanov, and Y. Kivshar, Phys. Rev. Lett. 121, 193903 (2018).

[16] A. Tittl, A. Leitis, M. Liu, F. Yesilkoy, D. Y. Choi, D. N. Neshev, Y. S. Kivshar, and H. Altug, Science 360, 1105 (2018).

[17] C. Jansen, I. A. I. Al-Naib, N. Born, and M. Koch, Appl. Phys. Lett. 98, 051109 (2011).

[18] S. Campione et al., ACS Photonics 3, 2362 (2016).

[19] S. I. Azzam, K. Chaudhuri, V. M. Shalaev, A. Boltasseva, and A. V. Kildishev, Proceedings of the 2019 Conference on Lasers and Electro-Optics (CLEO) (San Jose, CA, USA, May 5-10, 2019), 18863626

[20] J. Jeong, M. D. Goldflam, S. Campione, J. L. Briscoe, P. P. Vabishchevich, J. Nogan, M. B. Sinclair, T. S. Luk, and I. Brener, ACS Photonics 7, 1699 (2020).

[21] A. F. Oskooi, D. Roundy, M. Ibanescu, P. Bermel, J. D. Joannopoulos, and S. G. Johnson, Comput. Phys. Commun. 181, 687 (2010).

[22] U. Fano, Phys. Rev. 124, 1866 (1961).

[23] V. Savinov, V. A. Fedotov, and N. I. Zheludev, Phys. Rev. B 89, 205112 (2014). 